\title{
Biocompatibility and Antibacterial Effects of 6-Deoxy-6-Aminoethyleneamino Cellulose
}

\author{
Susanne Finger ${ }^{1}$, Michael Zieger ${ }^{1}$, Cornelia Wiegand1, Tim Liebert ${ }^{2}$, Thomas Heinze ${ }^{2}$, \\ Peter Elsner ${ }^{1}$, Uta-Christina Hipler ${ }^{1}$ \\ ${ }^{1}$ Department of Dermatology, University Medical Center, Jena, Germany \\ ${ }^{2}$ Center of Excellence for Polysaccharide Research, Institute of Organic Chemistry and Macromolecular Chemistry, \\ Friedrich Schiller University, Jena, Germany \\ Email: c.wiegand@med.uni-jena.de
}

How to cite this paper: Finger, S., Zieger, M., Wiegand, C., Liebert, T., Heinze, T., Elsner, P. and Hipler, U.-C. (2018) Biocompatibility and Antibacterial Effects of 6-Deoxy-6-Aminoethyleneamino Cellulose. Journal of Biosciences and Medicines, 6 , $51-62$.

https://doi.org/10.4236/jbm.2018.61006

Received: August 18, 2017

Accepted: January 7, 2018

Published: January 10, 2018

Copyright $\odot 2018$ by authors and Scientific Research Publishing Inc. This work is licensed under the Creative Commons Attribution International License (CC BY 4.0).

http://creativecommons.org/licenses/by/4.0/

\begin{abstract}
Recently, there is a need of alternatives to antibiotics due to increasing antibiotic-resistant microorganism. Promising classes of bioactive polymers are 6-deoxy-6-amino cellulose derivatives. The purpose of the study was the assessment of the biocompatibility of 6-deoxy-6-aminoethyleneamino cellulose (AEAC) with different degree of substitution (DS). HaCaT keratinocyte cell viability was analyzed by measuring the cellular ATP content. The antibacterial activity against Staphylococcus aureus and Klebsiella pneumoniae was examined by microplate laser nephelometry. Thus, the ratio of half-maximal lethal concentration $\left(\mathrm{LC}_{50}\right)$ and half-maximal inhibitory concentration $\left(\mathrm{IC}_{50}\right)$ was calculated and described as biocompatibility index. The study revealed that biocompatibility of AEAC depends on the DS. AEAC of low DS (0.3) showed the best biocompatibility.
\end{abstract}

\section{Keywords}

6-Deoxy-6-Aminoethyleneamino Celluloses, Antibacterial, Bacteria, Biocompatibility, Biomacromolecule, Keratinocyte

\section{Introduction}

There are different ways to deal with bacterial infections. To prevent or to treat bacterial infections antiseptics, disinfectants, antimicrobial peptides, or antibiotics are the means of choice. However, the latter are intensely discussed because frequent use of antibiotics leads to an increase of antibiotic resistance in bacteria. Thus, there is a need for alternatives. Antimicrobial polymers are considered to be valid options for commonly used products [1] [2] [3] [4] [5]. A choice could 
be the use of chemically modified cellulose such as 6-deoxy-6-amino cellulose derivatives. A typical example of this novel class of cellulose derivatives is 6-deoxy-6-aminoethyleneamino cellulose (AEAC) [3] [4] [6]. AEAC is described to be biocompatible [2] and hemocompatible [7].

AEACs are synthesized by nucleophilic displacement reaction of p-toluenesulfonic acid ester of cellulose with ethylene diamine [4] [7] [8] introducing amino moieties into the cellulose backbone that are water-soluble and may be positively charged depending on the $\mathrm{pH}$ value of the system [4]. The number of functional groups generated in the cellulose derivative is described as degree of substitution (DS) [9]. It is proposed that the functionalization with amino groups leads to antimicrobial activity. Besides antimicrobial efficacy, cytocompatibility is most important for the use of biomaterials in medical applications. Biocompatibility has become the central request for the medical application of materials and devices [10] [11] [12] [13]. Müller and Kramer defined a biocompatibility index (BI) for antiseptics based on the damage of murine fibroblasts and the MIC values for S. aureus and E. coli [14]. Following their approach [14], the BI was used to rate antibacterial activity of AEAC and effects on human cells. Here, the BI was defined as the ratio of the half-maximal lethal concentration $\left(\mathrm{LC}_{50}\right)$ and the half-maximal inhibitory concentration $\left(\mathrm{IC}_{50}\right)$. Accordingly, a $\mathrm{BI}$ greater than 1 will describe a substance exhibiting a high antimicrobial activity combined with a relatively low cytotoxicity (Figure 1(a)), whereas a BI less than 1 will indicate an antimicrobial effective sample with a relatively high cytotoxicity (Figure $1(b))$.

The aim of this study was the assessment of biocompatibility of AEAC with different DS. With regard to cytocompatibility, $\mathrm{HaCaT}$ keratinocytes have been used as a model system to determine cell viability by measuring cellular ATP content. S. aureus and $K$. pneumoniae were used as model organisms to investigate antibacterial activity of AEAC using microplate laser nephelometry (MLN). The ratio of half-maximal lethal concentration $\left(\mathrm{LC}_{50}\right)$ and half-maximal inhibitory

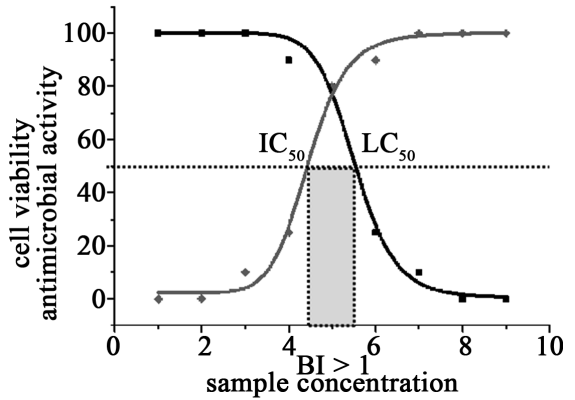

(a)

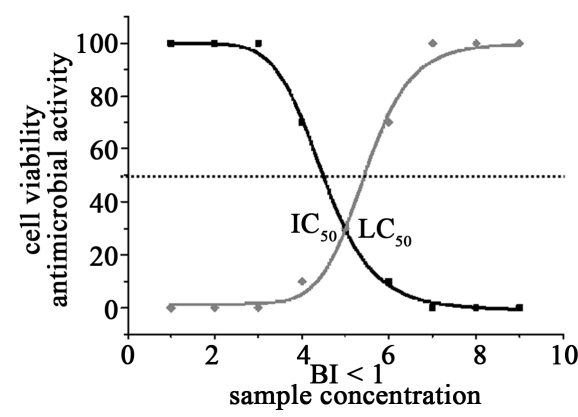

(b)

Figure 1. The biocompatibility index (BI) is composed of the ratio of half maximal lethal concentration $\left(\mathrm{LC}_{50}\right)$ to half maximal inhibitory concentration $\left(\mathrm{IC}_{50}\right)$. A BI greater than 1 indicates a substance with a high antimicrobial activity and good cell compatibility. In contrast a BI less than 1 indicates indeed a high antimicrobial activity but more than $50 \%$ of the human cells are death. The dotted line marks cell viability or antimicrobial activity at $50 \%$. 
concentration $\left(\mathrm{IC}_{50}\right)$ was calculated and described as biocompatibility index (BI). In addition, we determined the effect of DS on cytocompatibility and antimicrobial activity.

\section{Materials and Methods}

\subsection{Synthesis of 6-Deoxy-6-Aminoethyleneamino Cellulose}

Water-soluble 6-deoxy-6-aminoethyleneamino cellulose (AEAC, Figure 2) was synthesized by nucleophilic displacement reaction of cellulose tosylate as described elsewhere [2] [7]. AEAC with different degree of substitution (DS) of 0.3, $0.5,0.7$, and 0.9 were prepared. The initial AEAC concentration was $10 \mathrm{mg} / \mathrm{mL}$. Serial AEAC dilutions were prepared with respective cultivation medium.

\subsection{Cultivation of HaCaT Keratinocytes}

Human HaCaT keratinocytes (kindly provided by N. E. Fusenig, German Cancer Research Center Heidelberg, Germany) were cultured at $37^{\circ} \mathrm{C}$ in a humidified atmosphere containing 5\% $\mathrm{CO}_{2}$ in Dulbecco's modified Eagle's medium (Promocell, Germany) supplemented with $1 \%$ antibiotic-antimycotic solution (Promocell, Germany) and $10 \%$ fetal bovine serum (Promocell, Germany) as described elsewhere [15].

\subsection{Determination of Cell Viability}

HaCaT keratinocytes were incubated with AEAC dilutions for $24 \mathrm{~h}$ and afterwards cell viability was determined using a luminometric adenosine triphosphate (ATP) assay (ATPLite Kit; Perkin Elmer Life Sciences, Belgium) according to the manufacturer's instructions. The ATP-dependent light generation was measured using a microplate luminometer (LUMIstar Galaxy; BMG Labtech, Germany). The total amount of cellular ATP was measured after the cells were lysed. ATP concentration, calculated on the basis of a standard curve, is proportional to the number of metabolic active cells. Cell numbers were therefore calculated according to an ATP-cell number-standard curve [15]. Cells cultured in medium alone served as negative control. Triton-X100 (0.01\%) was used as a positive control for cytotoxicity. For evaluation, cellular proliferation under test conditions was expressed as percentage of the negative control. Half-maximal lethal concentration $\left(\mathrm{LC}_{50}\right)$ was calculated. Each substance concentration was

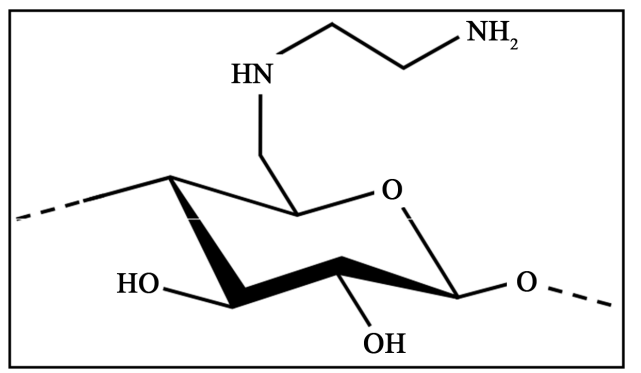

Figure 2. Schematic representation of 6-deoxy-6-aminoethyleneamino cellulose. 
tested in eightfold, and all experiments were performed at least twice.

\subsection{Cultivation of Bacteria}

Staphylococcus aureus ATCC 6538 and Klebsiella pneumoniae ATCC 4352 were purchased from the DSMZ (Deutsche Sammlung von Mikroorganismen und Zellkulturen, Germany). Strain maintenance was performed on Columbia agar plates with $5 \%$ sheep blood (Biomeriéux, France). Bacterial suspensions were prepared in CASO medium (Oxoid, UK) according to Finger et al. [16].

\subsection{Determination of Antibacterial Activity}

Effects of AEAC on $S$. aureus and $K$. pneumoniae were determined by microplate laser nephelometry (MLN) as previously reported [16] [17] [18] [19]. For this, bacterial suspensions $\left(\left(5 \times 10^{2}-7 \times 10^{2}\right.\right.$ colony forming units $)$ were incubated with AEAC dilutions in a microplate laser nephelometer (NEPHELOstar Galaxy; BMG Labtech, Germany) for $24 \mathrm{~h}$ at $37^{\circ} \mathrm{C}$. CASO medium alone was used as negative control and Chlorhexidine digluconate $(0.05 \%)$ as a positive control for antibacterial activity. The half-maximal inhibitory concentration $\left(\mathrm{IC}_{50}\right)$ of the AEAC under the experimental conditions used was calculated from the growth curves over $24 \mathrm{~h}$.

\subsection{Data Analysis and Statistics}

Experiments and measurements were performed in duplicate each. All values are expressed as means $\pm \mathrm{SE}$. $\mathrm{LC}_{50}$ and $\mathrm{IC}_{50}$ values were calculated using a logistic fit function as described elsewhere [5]

$$
y=\frac{A_{2}+\left(A_{1}-A_{2}\right)}{\left(1+\left[\frac{x}{x_{0}}\right]^{p}\right)}
$$

with $A_{1}=$ upper limit, $A_{2}=$ lower limit, $x_{0}=\mathrm{LC}_{50}$ or $\mathrm{IC}_{50}, p=$ slope of the curve; Origin 7.5 , USA.

The BI was calculated from the $\mathrm{LC}_{50}$ and $\mathrm{IC}_{50}$ values obtained after $24 \mathrm{~h}$.

$$
\mathrm{BI}=\frac{\mathrm{LC}_{50}}{\mathrm{IC}_{50}}
$$

\section{Results}

\subsection{Effects of AEAC on HaCaT Keratinocyte's Cell Viability}

For assessment of AEAC with regard to its effect on viability of HaCaT keratinocytes, cellular ATP content was determined using a luminometric assay. Because ATP can only be determined in living cells, information on cell viability is obtained. Hence, $\mathrm{HaCaT}$ keratinocytes were incubated with dissolved AEAC of different DS over $24 \mathrm{~h}$. In general, the AEAC of different DS were compatible to the $\mathrm{HaCaT}$ cells. However, $\mathrm{LC}_{50}$ values showed a distinct tendency; cytocompatibility decreased with increasing DS. The AEAC with the low DS of 0.3 showed 
the highest cytocompatibility, whereas the AEAC with the highest DS (0.9) was less cytocompatible (Table 1). Figure 3 shows the dependence of HaCaT cell viability on concentration and DS of the AEAC samples. Already a concentration of $39 \mu \mathrm{g} / \mathrm{mL}$ showed a distinct difference on HaCaT cell viability. AEAC with DS higher 0.5 exhibited a reduction in cell viability of 33\% (AEAC-DS0.7) or 38\% (AEAC-DS0.9) compared to the control. In case of AEAC with DS of 0.7 , a distinct decline in cell viability was observed at a concentration of 625 $\mu \mathrm{g} / \mathrm{mL}$. For the AEAC with low DS of 0.3 , cell viability is influenced at concentration higher than $2500 \mu \mathrm{g} / \mathrm{mL}$ (data not shown).

\subsection{Antibacterial Activity of AEAC}

The growth of $S$. aureus and $K$. pneumoniae under the influence of AEAC was monitored by MLN (Figure 4). It was found that the AEAC had bactericidal effects. It was shown that the antibacterial activity of AEAC depended on the DS. The $\mathrm{IC}_{50}$ values for both bacteria (Table 1) illustrate an increase of antibacterial

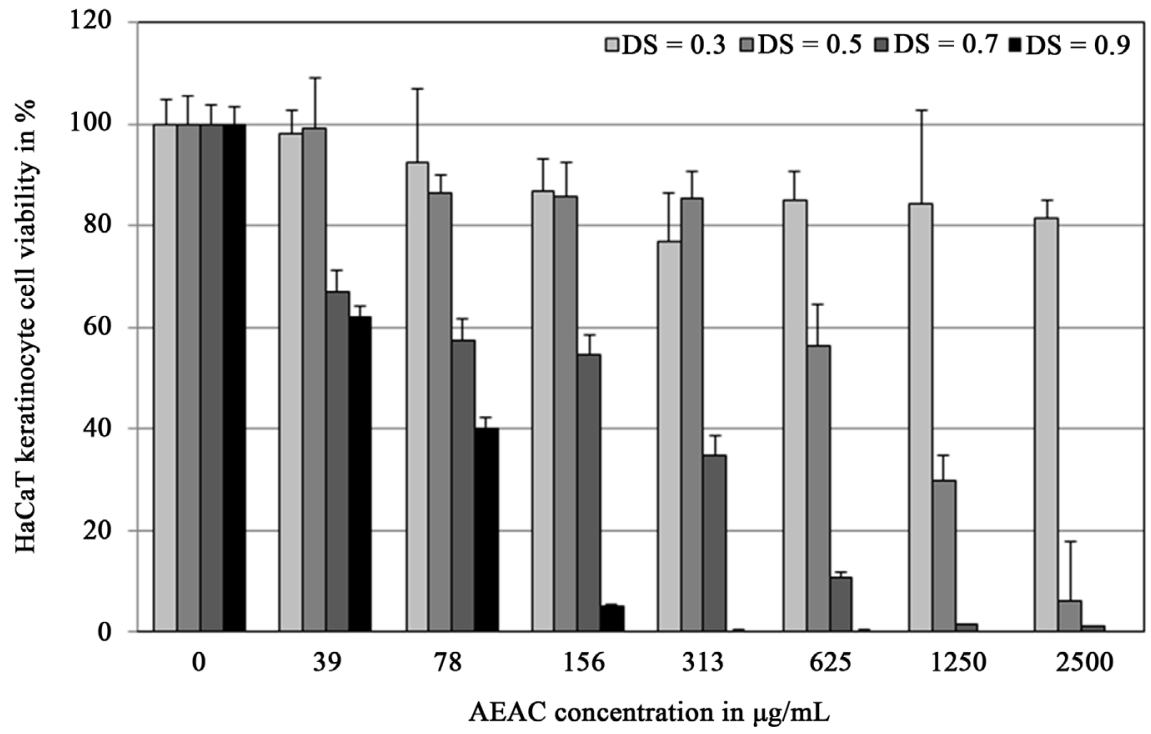

Figure 3. Determination of cell viability by measurement of the cellular ATP content after $24 \mathrm{~h}$ incubation of HaCaT keratinocytes with the different AEAC. AEAC exhibited concentration- and DS-dependent effects on HaCaT keratinocytes.

Table $1 . \mathrm{LC}_{50}$ and $\mathrm{IC}_{50}$ values were calculated by means of cell viability measurements and growth curves. The biocompatibility index (BI) was calculated by means of the ratio of $\mathrm{LC}_{50}$ and $\mathrm{IC}_{50}$ values.

\begin{tabular}{|c|c|c|c|c|c|}
\hline $\mathrm{DS}_{\mathrm{AEAC}}$ & $\begin{array}{c}\mathrm{LC}_{50} \text { in } \mu \mathrm{g} / \mathrm{mL} \\
\mathrm{HaCaT} \text { cells }\end{array}$ & $\begin{array}{c}\mathrm{IC}_{50} \text { in } \mu \mathrm{g} / \mathrm{mL} \\
S . \text { aureus }\end{array}$ & $\begin{array}{c}\mathrm{BI}\left(\mathrm{LC}_{50} / \mathrm{IC}_{50}\right) \\
\text { S. aureus }\end{array}$ & $\begin{array}{l}\mathrm{IC}_{50} \text { in } \mu \mathrm{g} / \mathrm{mL} \\
K \cdot \text { pneumoniae }\end{array}$ & $\begin{array}{l}\mathrm{BI}\left(\mathrm{LC}_{50} / \mathrm{IC}_{50}\right) \\
K \cdot \text { pneumoniae }\end{array}$ \\
\hline 0.3 & $3916.22 \pm 35.04$ & $90.21 \pm 7.93$ & 43 & $83.00 \pm 1.27$ & 47 \\
\hline 0.5 & $858.29 \pm 8.78$ & $42.36 \pm 0.79$ & 20 & $192.17 \pm 7.59$ & 4 \\
\hline 0.7 & $197.71 \pm 33.64$ & $17.91 \pm 2.41$ & 11 & $132.79 \pm 17.66$ & 1 \\
\hline 0.9 & $58.87 \pm 2.02$ & $20.60 \pm 0.29$ & 3 & $60.95 \pm 6.43$ & 1 \\
\hline
\end{tabular}




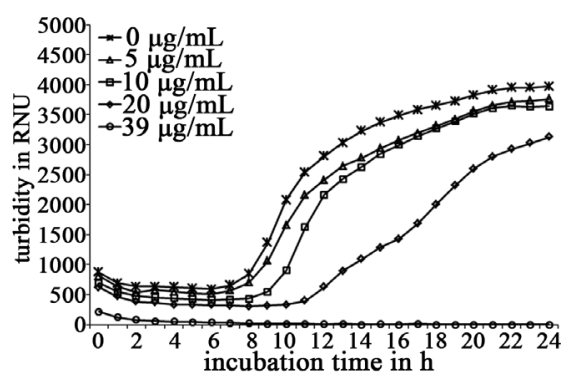

(a)

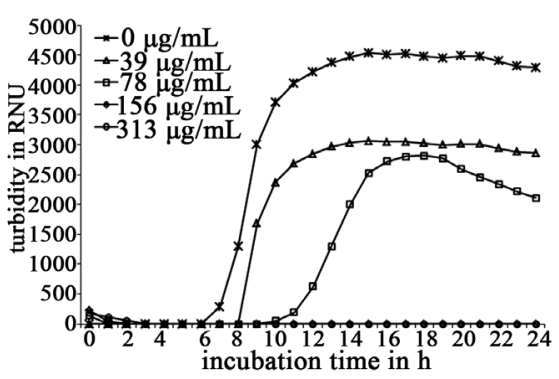

(b)

Figure 4. Growth curves determined by MLN over 24 h. Effects of AEAC-DS0.9 on $S$. aureus (a) and $K$. pneumoniae (b).

activity with increasing DS with the exception of AEAC with DS 0.3 and $K$. pneumoniae. The AEAC with the highest DS of 0.9 showed the strongest antibacterial effects, while the AEAC with the lowest DS of 0.3 was less effective against the bacteria. In general, AEAC were more effective against $S$. aureus compared to $K$. pneumoniae. For instance, $50 \%$ of $S$. aureus were inhibited with $20 \mu \mathrm{g} / \mathrm{mL}$ of AEAC with DS of 0.9 , whereas threefold higher concentrations were needed to achieve the same effects on $K$. pneumoniae. In case of AEAC with DS of 0.7 , the $\mathrm{IC}_{50}$ for $K$. pneumoniae $(132.79 \mu \mathrm{g} / \mathrm{mL})$ is almost seven times higher than for $S$. aureus $(17.91 \mu \mathrm{g} / \mathrm{mL})$. This trend was also observed for AEAC with DS of 0.5 .

\subsection{Biocompatibility of AEAC}

The results of the cell viability assay and MLN were summarized as the ratio from $\mathrm{LC}_{50}$ to $\mathrm{IC}_{50}$ to determine the biocompatibility of AEAC. The value is referred to as biocompatibility index (BI). A BI greater than 1 indicates a substance with a high antimicrobial activity at the same concentration where more than $50 \%$ of the human cells are still viable in vitro, while, a BI less than 1 indicates a high antimicrobial activity, but a low cytocompatibility. This study demonstrated that the BI depends on the DS of AEAC. AEAC with the lowest DS of 0.3 was most compatible to HaCaT keratinocytes. The cytocompatibility decreases with increasing DS. This also applies to the determined BI (Table 1). Thus, the cytocompatibility for AEAC with low DS is better than for AEAC with higher DS (Figure 5). The highest BI from about 40 ( $S$. aureus) and 50 (K. pneumoniae) could be calculated for AEAC with DS of 0.3 (Table 1). In addition, the BI determined with $S$. aureus was always higher than in connection with $K$. pneumoniae (Figure 5). Nonetheless, the BI was greater than or nearly 1 for every AEAC tested.

\section{Discussion}

Four AEAC with different DS were examined with regard to cytocompatibility and antibacterial activity. HaCaT keratinocytes were chosen to study the effects of AEAC on cell viability. This spontaneously immortalized cell line [20] is a 


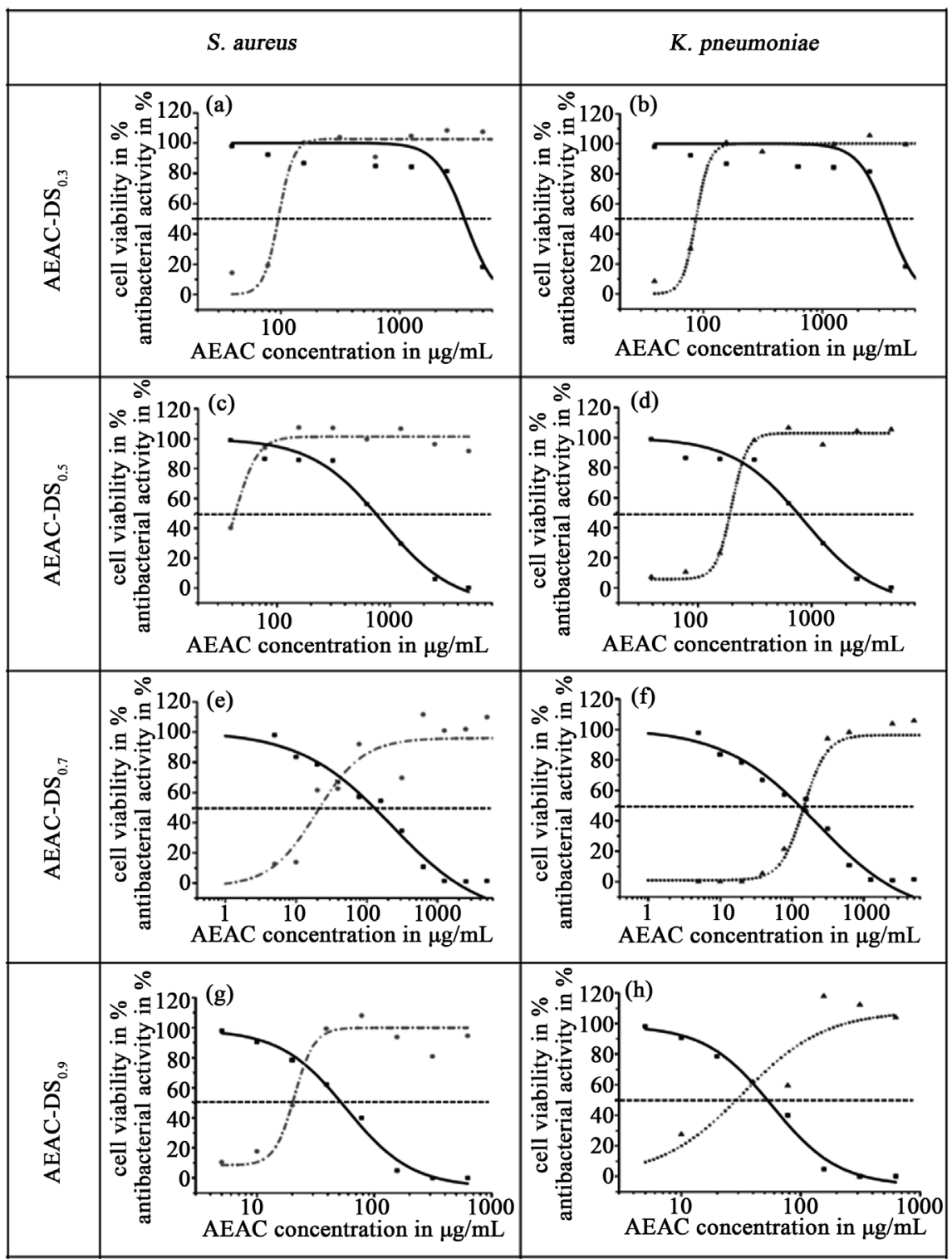

Figure 5. Effects of AEAC differ in DS on human HaCaT keratinocytes and on the bacteria $S$. aureus and $K$. pneumoniae. Graphs ((a)-(h)) demonstrate dose-responsecurves for cell viability (presented in black) or antibacterial activity (presented in grey), while the dotted line marks $50 \%$ of cell viability or antibacterial activity. With increasing DS cell viability declined and antibacterial activity increased, except of AEAC-DS0.3 and K. pneumoniae.

suitable model to determine effects of diverse substances on cytology in vitro [10]. The antibacterial activity was monitored with respect to the bacteria $S$. aureus und $K$. pneumoniae by MLN. As described previously, MLN is a sensitive and suitable method to determine the influence of substances on microbial growth [17] [21] [22] [23]. To evaluate biocompatibility of AEAC, the ratio of $\mathrm{LC}_{50}$ and $\mathrm{IC}_{50}$ was calculated and referred to as biocompatibility index.

In consideration of the effects of AEAC on cell viability of HaCaT keratinocytes, it appears that the DS plays an important role. Thus, $\mathrm{LC}_{50}$ values decreased with 
increasing DS. At low DS of AEAC, the effects on HaCaT keratinocytes were minimal. A decline in cell viability was observed with increasing DS. AEAC can be compared to chitosan to a certain extend; it appears to be a suitable synthetic version of chitosan. For chitosan it was reported to interact with negative charges in the skin [24]. He et al. found that chitosan and its derivatives could significantly change the secondary structure of keratin and increase the water content in Stratum corneum of skin, decrease $\mathrm{HaCaT}$ cell membrane potential and enhance cell membrane fluidity to various degrees [25]. The mode of action of chitosan leads to the suggestion that the positively charged AEAC substituents possibly interact with components of cell membranes or with components inside cells and this might lead to cytotoxic effects. However, the mode of action is not fully understood yet [26] [27]. The mode of action of AEAC suggested requires an examination of hemocompatibility for medical applications with tight contact to tissue and blood. Zieger et al. also tested AEAC with different DS in regard to hemolysis and markers for coagulation in human whole blood, human platelet rich plasma, human pooled plasma and erythrocytes suspensions [7]. It was described that AEAC showed concentration- and DS-dependent influence on hemocompatibility in vitro. Thus, AEAC with low DS had less influence on blood coagulation, a low hemolytic effect, and provided the highest hemocompatibility.

As for the biocompatibility of AEAC, it is crucial to determine antibacterial activity besides the cytocompatibility. The antimicrobial activity of chitosan was frequently mentioned in literature [19] [28] [29] [30] and could give some hints for understanding the effects of AEAC on microorganism. Hosseinnejad and Jafari reviewed different factors and mechanisms, which play a role in antimicrobial activity of chitosan [31]. For instance, molecular weight, degree of acetylation, concentration, and $\mathrm{pH}$ value as well as microbial species are named as antimicrobial properties.

In the present study, the differences of antibacterial efficacy observed against $S$. aureus and $K$. pneumoniae of AEAC possibly depend on the morphological composition of bacterial cell walls. AEACs were most effective against the Gram-positive bacterium $S$. aureus. For $K$. pneumoniae, a Gram-negative bacterium, higher AEAC concentrations were needed to inhibit bacterial growth compared to $S$. aureus. Hence, $S$. aureus was more sensitive to AEAC than $K$. pneumoniae. Jou also described comparable observation when $S$. aureus and $K$. pneumoniae were incubated with the chitosan derivative chitosan- $\mathrm{N}$-hydroxy2,3-propyl-N-methyl-N,N-diallylammonium methyl sulfate [28]. On the contrary to Gram-positive bacteria, Gram-negative bacteria possess a supplementary barrier in form of the lipopolysaccharide layer [16] [32] [33], which explains why Gram-negative are less sensitive.

In case of the AEAC with DS of 0.3 , the determined $\mathrm{IC}_{50}$ values for both bacteria showed no distinctly difference in antibacterial activity (S. aureus: $90 \mu \mathrm{g} / \mathrm{mL}$ and $K$. pneumoniae: $83 \mu \mathrm{g} / \mathrm{mL}$ ). A reason for this observation could be the fact that AEAC with low DS have the property to agglutinate surfaces [2] [34]. This 
effect declines of samples of higher DS. Therefore, at DS higher 0.3 differences in antibacterial activity were observed with regard to cell wall composition. Similar to the antibacterial activity of AEAC with DS of 0.3 , the BI for $S$. aureus and $K$. pneumoniae does not differ. Due to the sensitivity of $S$. aureus against AEAC, the $\mathrm{BI}$ are higher than for $K$. pneumoniae. With regard to the effects of AEAC on cell viability of $\mathrm{HaCaT}$ keratinocytes and on both bacteria, it is apparent that concentrations at which $S$. aureus was killed no effects on HaCaT cell viability were observed. That was the case for AEAC with DS of up to 0.5. AEAC with a DS above 0.5 showed only slight effects on cell viability by simultaneous killing of $S$. aureus. For $K$. pneumoniae this observation only applies to AEAC with DS of 0.3. With increasing DS, AEAC exhibited cytotoxic effects on HaCaT cells before growth of $K$. pneumoniae was inhibited completely that is also caused by less sensitivity of Gram-negative bacteria to antimicrobials as already mentioned.

It was found that AEAC with low DS $(\leq 0.5)$ had less effect on cell viability of $\mathrm{HaCaT}$ keratinocytes than AEAC with higher DS (>0.5). Cell viability declined and antibacterial activity increased with increasing DS, except of AEAC-DS0.3 and $K$. pneumoniae. Whereat, $S$. aureus was more sensitive to the tested AEAC than $K$. pneumoniae.

AEAC with a maximum DS of 0.5 are most recommendable for medical applications because they showed the highest BI of all tested AEAC. Zieger et al. came to the same conclusion and recommend AEAC with DS of 0.5 for applications that require inert materials [7]. AEAC with low DS exhibited the lowest anticoagulant and hemolytic potential of AEAC tested. In addition to AEAC solutions, also applications of corresponding nanoparticles are possible [4] [6].

\section{Acknowledgements}

The authors would like to thank Martina Grebner and Peggy Laudely for technical assistance. This study was kindly funded by the Federal Ministry of Education and Research (03WKP16B).

\section{References}

[1] Siedenbiedel, F. and Tiller, J.C. (2012) Antimicrobial Polymers in Solution and on Surfaces: Overview and Functional Principles. Polymers, 4, 46-71.

https://doi.org/10.3390/polym4010046

[2] Heinze, T., Liebert, T., Miethe, P., Schlufter, K., Hipler, U.C. and Wiegand, C. (2015) Di- and Multifunctional Amine-Modified Oligo- and Polysaccharide Derivatives as Anti-Infective Substances and the Use Thereof. WO 2013132061 A1.

[3] Roemhild, K., Wiegand, C., Hipler, U.C. and Heinze, T. (2013) Novel Bioactive Amino-Functionalized Cellulose Nanofibers. Macromolecular Rapid Communications, 34, 1767-1771. https://doi.org/10.1002/marc.201300588

[4] Wiegand, C., Nikolajski, M., Hipler, U.C. and Heinze, T. (2015) Nanoparticle Formulation of AEA and BAEA Cellulose Carbamates Increases Biocompatibility and Antimicrobial Activity. Macromolecular Bioscience, 15, 1242-1251.

https://doi.org/10.1002/mabi.201500031 
[5] Wiegand, C., Bauer, M., Hipler, U.C. and Fischer, D. (2013) Poly(ethyleneimines) in Dermal Applications: Biocompatibility and Antimicrobial Effects. International Journal of Pharmaceutics, 456, 165-174. https://doi.org/10.1016/j.ijpharm.2013.08.001

[6] Nikolajski, M., Wotschadlo, J., Clement, J.H. and Heinze, T. (2012) Amino-Functionalized Cellulose Nanoparticles: Preparation, Characterization, and Interactions with Living Cells. Macromolecular Bioscience, 12, 920-925. https://doi.org/10.1002/mabi.201200040

[7] Zieger, M., Wurlitzer, M., Wiegand, C., Reddersen, K., Finger, S., Elsner, P., Laudeley, P., Liebert, T., Heinze, T. and Hipler, U.C. (2015) 6-Deoxy-6-aminoethyleneamino Cellulose: Synthesis and Study of Hemocompatibility. Journal of Biomaterials Science, Polymer Edition, 26, 931-946. https://doi.org/10.1080/09205063.2015.1068546

[8] Tiller, J., Berlin, P. and Klemm, D. (1999) A Novel Efficient Enzyme-Immobilization Reaction on $\mathrm{NH}_{2}$ Polymers by Means of L-Ascorbic Acid. Biotechnology and Applied Biochemistry, 30, 155-162.

[9] Vigo, T.L. and Sachinvala, N. (1999) Deoxycelluloses and Related Structures. Polymers for Advanced Technologies, 10, 311-320.

https://doi.org/10.1002/(SICI)1099-1581(199906)10:6<311::AID-PAT880>3.0.CO;2$\underline{\mathrm{G}}$

[10] Wiegand, C., Heinze, T., Hipler, U. C. (2009) Comparative in Vitro Study on Cytotoxicity, Antimicrobial Activity, and Binding Capacity for Pathophysiological Factors in Chronic Wounds of Alginate and Silver-Containing Alginate. Wound Repair and Regeneration, 17, 511-521. https://doi.org/10.1111/j.1524-475X.2009.00503.x

[11] Marques, A.P., Reis, R.L. and Hunt, J.A. (2002) The Biocompatibility of Novel Starch-Based Polymers and Composites: In Vitro Studies. Biomaterials, 23, 1471-1478. https://doi.org/10.1016/S0142-9612(01)00272-1

[12] Kirkpatrick, C.J., Bittinger, F., Wagner, M., Kohler, H., van Kooten, T.G., Klein, C. L. and Otto, M. (1998) Current Trends in Biocompatibility Testing. Proceedings of the Institution of Mechanical Engineers, Part H, 212, 75-84. https://doi.org/10.1243/0954411981533845

[13] Ř́hová, B. (1996) Biocompatibility of Biomaterials: Hemocompatibility, Immunocompatiblity and Biocompatibility of Solid Polymeric Materials and Soluble Targetable Polymeric Carriers. Advanced Drug Delivery Reviews, 21, 157-176. https://doi.org/10.1016/S0169-409X(96)00404-8

[14] Müller, G. and Kramer, A. (2008) Biocompatibility Index of Antiseptic Agents by Parallel Assessment of Antimicrobial Activity and Cellular Cytotoxicity. Journal of Antimicrobial Chemotherapy, 61, 1281-1287. https://doi.org/10.1093/jac/dkn125

[15] Wiegand, C., Winter, D. and Hipler, U.C. (2010) Molecular-Weight-Dependent Toxic Effects of Chitosans on the Human Keratinocyte Cell Line HaCaT. Skin Pharmacology and Physiology, 23, 164-170. https://doi.org/10.1159/000276996

[16] Finger, S., Wiegand, C., Buschmann, H.J. and Hipler, U.C. (2013) Antibacterial Properties of Cyclodextrin-Antiseptics-Complexes Determined by Microplate Laser Nephelometry and ATP Bioluminescence Assay. International Journal of Pharmaceutics, 452, 188-193. https://doi.org/10.1016/j.ijpharm.2013.04.080

[17] Finger, S., Wiegand, C., Buschmann, H.J. and Hipler, U.C. (2012) Antimicrobial Properties of Cyclodextrin-Antiseptics-Complexes Determined by Microplate Laser Nephelometry and ATP Bioluminescence Assay. International Journal of Pharmaceutics, 436, 851-856. https://doi.org/10.1016/j.ijpharm.2012.07.009 
[18] Wiegand, C., Abel, M., Ruth, P. and Hipler, U.C. (2012) Analysis of the Adaptation Capacity of Staphylococcus aureus to Commonly Used Antiseptics by Microplate laser Nephelometry. Skin Pharmacology and Physiology, 25, 288-297. https://doi.org/10.1159/000341222

[19] Seyfarth, F., Schliemann, S., Elsner, P. and Hipler, U.C. (2008) Antifungal Effect of High- and Low-Molecular-Weight Chitosan Hydrochloride, Carboxymethyl Chitosan, Chitosan Oligosaccharide and N-Acetyl-D-Glucosamine against Candida albicans, Candida krusei and Candida glabrata. International Journal of Pharmaceutics, 353, 139-148.

[20] Boukamp, P., Petrussevska, R.T., Breitkreutz, D., Hornung, J., Markham, A. and Fusenig, N.E. (1988) Normal Keratinization in a Spontaneously Immortalized Aneuploid Human Keratinocyte Cell Line. The Journal of Cell Biology, 106, 761-771. https://doi.org/10.1083/jcb.106.3.761

[21] Fouda, M.M., Knittel, D., Hipler, U.C., Elsner, P. and Schollmeyer, E. (2006) Antimycotic Influence of Beta-Cyclodextrin Complexes-In Vitro Measurements Using Laser Nephelometry in Microtiter Plates. International Journal of Pharmaceutics, 311, 113-121. https://doi.org/10.1016/j.ijpharm.2005.12.028

[22] Joubert, A., Calmes, B., Berruyer, R., Pihet, M., Bouchara, J.P., Simoneau, P. and Guillemette, T. (2010) Laser Nephelometry Applied in an Automated Microplate System to Study Filamentous Fungus Growth. Biotechniques, 48, 399-404. https://doi.org/10.2144/000113399

[23] Hipler, B., Brand, S., Angersbach, S. and Rückert, C. (2003) Monitoring des Wachstums von Mikroorganismen mit Hilfe der Nephelometrie. BIOspektrum, 9 , 648-649.

[24] Taveira, S.F., Nomizo, A. and Lopez, R.F. (2009) Effect of the Iontophoresis of a Chitosan Gel on Doxorubicin Skin Penetration and Cytotoxicity. Journal of Controlled Release: Official Journal of the Controlled Release Society, 134, 35-40. https://doi.org/10.1016/j.jconrel.2008.11.002

[25] He, W., Guo, X., Xiao, L. and Feng, M. (2009) Study on the Mechanisms of Chitosan and Its Derivatives Used as Transdermal Penetration Enhancers. International Journal of Pharmaceutics, 382, 234-243. https://doi.org/10.1016/j.ijpharm.2009.07.038

[26] Waschinski, C.J. and Tiller, J.C. (2005) Poly(oxazoline)s with Telechelic Antimicrobial Functions. Biomacromolecules, 6, 235-243. https://doi.org/10.1021/bm049553i

[27] Milovic, N.M., Wang, J., Lewis, K. and Klibanov, A.M. (2005) Immobilized N-alkylated Polyethylenimine Avidly Kills Bacteria by Rupturing Cell Membranes with No Resistance Developed. Biotechnology and Bioengineering, 90, 715-722. https://doi.org/10.1002/bit.20454

[28] Jou, C.H. (2011) Antibacterial Activity and Cytocompatibility of Chitosan-N-Hydroxy-2,3-Propyl-N methyl-N,N-diallylammonium Methyl Sulfate. Colloids and Surfaces. Biointerfaces, 88, 448-454. https://doi.org/10.1016/j.colsurfb.2011.07.028

[29] No, H.K., Park, N.Y., Lee, S.H. and Meyers, S.P. (2002) Antibacterial Activity of Chitosans and Chitosan Oligomers with Different Molecular Weights. International Journal of Food Microbiology, 74, 65-72. https://doi.org/10.1016/S0168-1605(01)00717-6

[30] Muzzarelli, R., Tarsi, R., Filippini, O., Giovanetti, E., Biagini, G. and Varaldo, P.E. (1990) Antimicrobial Properties of N-Carboxybutyl Chitosan. Antimicrobial Agents and Chemotherapy, 34, 2019-2023. https://doi.org/10.1128/AAC.34.10.2019 
[31] Hosseinnejad, M. and Jafari, S.M. (2016) Evaluation of Different Factors Affecting Antimicrobial Properties of Chitosan. International Journal of Biological Macromolecules, 85, 467-475. https://doi.org/10.1016/j.ijbiomac.2016.01.022

[32] Denyer, S.P. (1995) Mechanisms of Action of Antibacterial Biocides. International Biodeterioration \& Biodegradation, 36, 227-245.

https://doi.org/10.1016/0964-8305(96)00015-7

[33] Kenawy, E.-R., Worley, S.D. and Broughton, R. (2007) The Chemistry and Applications of Antimicrobial Polymers: A State-of-the-Art Review. Biomacromolecules, 8 , 1359-1384. https://doi.org/10.1021/bm061150q

[34] Heinze, T. (2015) Cellulose: Structure and Properties. Springer, Berlin Heidelberg, 1-52. 